\title{
Study of Biofilm Production and Antimicrobial Susceptibility Pattern in Clinical Isolates of Proteus Species at a Tertiary Care Hospital
}

\author{
R. Karthik*, R. Ambica and T. Nagarathnamma \\ Department of microbiology, Bangalore medical college and research institute, \\ Bangalore - 560002, Karnataka, India \\ *Corresponding author
}

\section{Keywords \\ Proteus species, Biofilm, Antibiotic susceptibility}

Article Info

\section{Accepted:}

06 December 2017

Available Online:

10 January 2018

\section{A B S T R A C T}

Proteus species are among the commonly implicated pathogens in hospital as well as community acquired infections. They cause nosocomial sinusitis, intra-abdominal abscesses, biliary tract infection, surgical site infection, soft tissue infection and osteomyelitis. They cause significant clinical infections, which are difficult to eradicate especially from hosts with wounds, catheterization, underlying diseases and in immunocompromised states. The therapeutic obstacles during Proteus species treatment can be connected with its ability to form biofilm, antibiotic resistance due to biofilm formation leading to persistence of infections. Hence the study is undertaken to find out the ability of Proteus species isolated from different clinical samples to form biofilm and their antibiotic susceptibility pattern. Total of 150 Proteus species isolates from different clinical samples such as urine, purulent material from wounds or abscesses, ear swabs, sputum, blood or aspirates (of joint fluid, pleural fluid, ascitic fluid and pus) collected from patients suspected of bacterial infection at Victoria Hospital, Vani Vilas Hospital, attached to Bangalore Medical College and Research Institute during the period November 2012November 2014. Samples were collected after taking informed consent from all patients and were processed. The identification of Proteus species was done using standard biochemical tests. Proteus species thus identified were screened for biofilm production by tissue culture Plate (TCP) method, tube adherence method and Congo red agar method and studied for the antibiotic susceptibility patterns by in-vitro testing by Kirby-Bauer disc diffusion method performed according to CLSI recommendations. Out of the 150 Proteus species isolated from various clinical samples, 79 were from male and 71 were from females. Majority of clinical samples from which Proteus species were isolated were from pus samples $(91.33 \%)$. Among them, majority were from patients of thermal burns $(34 \%)$. Proteus mirabilis was the most isolated species (57.3\%). For detection of biofilm, tissue culture plate method was considered as gold standard. Among the isolates, $48 \%$ were biofilm producers and $52 \%$ were non/weak biofilm producers. Among Proteus mirabilis, $52.32 \%$ were biofilm producers whereas among Proteus vulgaris, $42.59 \%$ were biofilm producers. Among the MDR isolates, $56.5 \%$ of the isolates showed biofilm production whereas $40(43.4 \%)$ of isolates were weak/non biofilm producers. Majority of Proteus isolates showed sensitivity to Imipenem $(81.1 \%)$, Piperacillin + Tazobactum (83\%). Sensitivity was highest with Congo red agar method and tube adherence methods in combination $(86.11 \%)$. Congo red agar method showed better specificity $82.05 \%$ than other methods. There is an increase in isolation of Proteus species from wounds than from urinary tract infections with significant numbers showing biofilm forming capability. Tissue culture plate method was considered as gold standard method for biofilm detection in the present study. Also biofilm forming Proteus species isolates showed more antimicrobial drug resistance. Multi drug resistant Proteus isolates showed more biofilm forming ability indicating need for further studies on Proteus species and their ability to form biofilms. Also further studies on better understanding of bacterial biofilm interactions at molecular level for this species are necessary as they are more frequently being implicated in chronic infections and treatment failures. Also Tube adherence method and Congo red agar method in combination proved very effective for screening biofilm forming isolates. Though PCR technique is the most accurate and specific method, it is not readily available in resource limited settings and the need of the hour is rapid, cheap, cost effective and easily available methods for detecting biofilm formation. In view of emerging drug resistance and multidrug resistance exhibited by Proteus species, periodic review and formulation of antibiotic policy are needed. Indiscriminate use of antibiotic must be discouraged and therapy should be advocated as for as possible after the culture and sensitivity reports are available. 


\section{Introduction}

Proteus species are among the commonly implicated pathogens in hospital as well as community acquired infections. (Mandell Gerald) The Proteus group belongs to the family of Enterobacteriaceae, the genus Proteus. Proteus species cause 5\% of cases of hospital-acquired (UTI) and are responsible for $10-15 \%$ of cases of complicated UTI, primarily those associated with catheterization (Longo Dan et al., 2011). P. mirabilis is the third most commonly isolated pathogen (after Escherichia coli and Klebsiella pneumoniae) of urinary tract infections. Among UTI isolates from chronically catheterized patients, the prevalence of Proteus is 20-45\% (Longo Dan et al., 2011). They also cause nosocomial sinusitis, intra-abdominal abscesses, biliary tract infection, surgical site infection, soft tissue infection and osteomyelitis.

The therapeutic obstacles during Proteus species treatment can be connected with its ability to form biofilm (Joanna KwiecińskaPiróg et al., 2013). Biofilms are bacterial populations that are enclosed in a matrix of extracellular polymeric substances (Mandell Gerald; Zubair et al., 2011; Stoodley et al., 2004). Biofilm forms on living (e.g. wounds) or non-living surfaces such as intravenous catheters, artificial joints, cardiac pacemakers and establishes a protective environment in natural, industrial and hospital settings. Biofilm-living bacteria are phenotypically different from their planktonic counterparts.

Biofilm forming cells exhibit antibiotic resistance to which their planktonic counterparts appear susceptible by in vitro testing. This is due to following reasons:

Nearness of cells within biofilm enhances the transfer of plasmids coding virulence factors and the mechanisms of antibiotic resistance.

Mucus and glycocalyx reduce antibiotic distribution into the deeper layers of the biofilm.

Quorum sensing (QS) enables transfer of information connected with biocidal agents' resistance and the mechanisms of their activation. Thus, biofilm forming bacteria are the cause of many chronic, recurrent and persistent infections (Stoodley et al., 2004).

Concept of bacterial biofilms has emerged as a potential way to better understand how bacteria deter healing. Also according to a public announcement from National Institute of Health (NIH), more than $60 \%$ of all infections are caused by biofilm forming bacteria (Zubair et al., 2011). Therefore, a better understanding of bacterial biofilms is needed, and this may ultimately result in development of novel therapeutics for the prevention and treatment. Hence the study is undertaken to find out the biofilm producers among Proteus species with their antibiotic susceptibility pattern.

\section{Aims and objectives of the study}

To isolate and identify Proteus species from various clinical specimens.

To determine the antibiotic susceptibility pattern of the isolates.

To determine biofilm producers among the isolates by tissue culture plate (TCP), Congo red agar and tube adherence methods and to compare these methods.

\section{Materials and Methods}

\section{Source of data}

This study was conducted from November 2012-November 2014. Different clinical samples such as urine, purulent material from wounds or abscesses, ear swabs, sputum, 
blood or aspirates (of joint fluid, pleural fluid, ascitic fluid and pus) collected from patients suspected of bacterial infection were processed by microscopy and culture.

A total of 150 isolates of Proteus species isolated from such cases were identified to species level and studied for their ability to form biofilm by three phenotypic methods and their antimicrobial susceptibility patterns were studied.

\section{Method of collection}

The study population included both out patients and in patients attending different clinical departments of Victoria and Vani Vilas hospitals, Bangalore Medical College and Research Institute (BMC\&RI). The specimens were transported to the laboratory within 2 hours and processed.

\section{Inclusion criteria}

Proteus species isolates from different clinical samples such as urine, purulent material from wounds or abscesses, ear swabs, sputum, blood or aspirates (of joint fluid, pleural fluid, ascitic fluid and pus) collected from patients suspected of bacterial infection.

\section{Exclusion criteria}

Any bacterial growth other than Proteus species.

Any fungal growth

\section{Laboratory procedures}

Clinical samples of pus, urine, sputum, blood and other samples sent to Microbiology laboratory, BMC \& RI were processed. The specimens were brought to the laboratory within $2 \mathrm{hrs}$ of collection for processing.

\section{Gram-stain}

Smears were made from all samples except blood, heat-fixed and stained by Gram-stain. Smears were examined for the presence of pus cells and gram-negative organisms.

\section{Culture}

Pus, sputum and other samples were inoculated onto MacConkey agar and Blood agar. The media were incubated aerobically overnight at $37^{\circ} \mathrm{C}$ and observed for growth on the next day. Blood samples were inoculated in blood culture bottles were incubated at $37^{\circ}$ $\mathrm{C}$ under aerobic conditions in the incubator for 7 days. The first subculture was done after 24 hours of incubation, the second on the third day and a final on the seventh day. On the next day it was inoculated onto MacConkey agar and Blood agar.

Urine sample was inoculated with standard loop on MacConkey agar [MA] and blood agar [BA]. After $24 \mathrm{hrs}$ of incubation at $37^{\circ} \mathrm{C}$, cultures were further processed. Cultures yielding no growth and insignificant/mixed growth were excluded.

Depending on the morphology of colonies, the presumptive identification of the organism was made (Collee et al., 1996):

Proteus species: On MA - Pale/ colorless colonies [non-lactose fermenters].

On BA - Colonies with characteristic swarming and fishy odour.

The isolates were bio-chemically identified by the following tests:

Proteus species: Oxidase non producer, Catalase producer, Methyl Red Positive, Vogues Proskauer variable, TSI agar showing $\mathrm{K} / \mathrm{A}$ with gas and with abundant $\mathrm{H}_{2} \mathrm{~S}$, Urease 
produced, Citrate variable utilization, phenyl alanine deaminase producer.

Proteus mirabilis: Indole non producer Ampicillin sensitive $(\geq 17 \mathrm{~mm})$, Ampicillin resistant $(\leq 13 \mathrm{~mm})$ and maltose non fermenter.

Proteus penneri: Indole non producer, Ampicillin resistant $(\leq 13 \mathrm{~mm})$ and maltose fermenter.

Proteus vulgaris: Indole producer, salicin fermenter

Proteus hauseri: Indole producer, salicin non fermenter

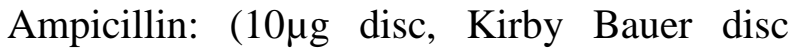
diffusion test as per CLSI guidelines)

$\begin{array}{llr}\text { Antimicrobial } & \text { susceptibility } & \begin{array}{r}\text { testing } \\ \text { (Clinical and } \\ \text { Laboratory }\end{array} \\ \text { Standard }\end{array}$

Antimicrobial susceptibility testing of isolates will be done according to Kirby-Bauer disc diffusion method on Mueller Hinton Agar (MHA) for Amikacin (30 $\mu$ g), Gentamicin $(10 \mu \mathrm{g})$, Ampicillin $(10 \mu \mathrm{g})$, Aztreonam $(30 \mu \mathrm{g})$, Cefotaxime $\quad(30 \mu \mathrm{g}), \quad$ Cotrimoxazole (Trimethoprim- sulfamethoxazole$1.25 / 23.75 \mu \mathrm{g})$, Norfloxacin $(10 \mu \mathrm{g})$ (for urine isolates) Ceftriaxone $(30 \mu \mathrm{g})$, Imipenem $(10 \mu \mathrm{g})$, Piperacillin/tazobactam $(100 / 10 \mu \mathrm{g})$. Interpretation will be done according to CLSI recommendations.

\section{Biofilm assay - Tissue Culture Plate (TCP) method}

The biofilm assay described by Zubair et al., (2011) is the first method adopted. Prior to biofilm production assay, the strains are cultured on Blood Agar and incubated overnight aerobically at $37^{\circ} \mathrm{C}$. After verifying purity of the tested strain, several colonies with identical morphology are suspended in sterile physiological saline. The turbidity of the bacterial suspension is adjusted to 0.5 of the McFarland standard $\left(\sim 1.5 \times 10^{8} \mathrm{CFU} / \mathrm{ml}\right)$. Then $10 \mathrm{ml}$ of trypticase soy broth (TSB) with $1 \%$ glucose is inoculated with a loopful of test organism from overnight culture on nutrient agar. The TSB broth is incubated at $37^{\circ} \mathrm{C}$ for 24 hours. The culture is further diluted 1:100 with fresh medium and flat bottom tissue culture plates (96 wells) are filled with $200 \mu 1$ of diluted cultures individually. Uninoculated sterile broth served as blank. Positive and negative controls were also inoculated. The culture plates are incubated at $37^{\circ} \mathrm{C}$ for 24 hours. After incubation, gentle tapping of the plates is done. The wells are washed with 200 $\mu \mathrm{l}$ of phosphate buffer saline $(\mathrm{pH}$ 7.2) four times to remove free-floating bacteria. Biofilms which remain adherent to the walls and the bottoms of the wells are fixed with absolute methanol and stained with $0.1 \%$ crystal violet. Excess stain is washed with deionized water and plates are dried properly. Optical densities (OD) of stained adherent biofilm are obtained with a micro ELISA auto-reader at wavelength of $570 \mathrm{~nm}$. Experiments were performed in triplicate. For all tested strains and negative controls, the interpretation of biofilm production was done according to the criteria of (Stepanovic et al., 2017).

The average OD values are calculated (from the inoculated triplets). The cut-off value (ODc) was established; the ODc is defined as three standard deviations (SD) above the mean $\mathrm{OD}$ of the negative control. The OD value of the tested strain is expressed as average OD value of the strain reduced by ODc value. ODc value should be calculated for each microtiter plate separately.

$\mathrm{ODc}=\varnothing \mathrm{OD}_{\text {negative control }}+3 \times \mathrm{SD}_{\text {negative control }}$ $\mathrm{OD}=\varnothing \mathrm{OD}_{\text {tested strain }}-\mathrm{OD}_{\mathrm{c}}$ $\varnothing=$ Average, $S D=$ Standard devaition 
For easier interpretation of the results, strains may be divided into the following categories: strain not producing biofilm, strain weakly producing biofilm, strain moderately producing biofilm and strain strongly producing biofilm. This categorization should be based of the previously calculated OD values (for this type of data interpretation the OD value of the strain should not be reduced by $\mathrm{OD}_{\mathrm{c}}$ value).

$\mathrm{OD} \leq \mathrm{OD}_{\mathrm{c}} \rightarrow$ Strain not producing biofilm

$\mathrm{OD}_{\mathrm{c}}<\mathrm{OD} \leq 2 \mathrm{x} \quad \mathrm{OD}_{\mathrm{c}} \rightarrow$ Strain weakly producing biofilm

$2 \mathrm{x} \mathrm{OD}_{\mathrm{c}}<\mathrm{OD} \leq 4 \mathrm{x} \mathrm{OD}_{\mathrm{c}} \rightarrow$ Strain moderately producing biofilm

$4 \mathrm{x} \quad \mathrm{OD}_{\mathrm{c}}<\mathrm{OD} \rightarrow$ Strain strongly producing biofilm

Biofilm production by using Congo Red Agar (CRA) medium

It is the second method done as described by Freeman et al., (1989) and will be the second method adopted. It is a qualitative method to detect biofilm production by using Congo Red Agar (CRA) medium. CRA medium is prepared with brain heart infusion broth 37 $\mathrm{g} / \mathrm{L}$, sucrose $50 \mathrm{~g} / \mathrm{L}$, agar $10 \mathrm{~g} / \mathrm{L}$ and Congo Red indicator $-0.8 \mathrm{~g} / \mathrm{L}$. First Congo red stain is prepared as a concentrated aqueous solution and autoclaved $\left(121^{\circ} \mathrm{C}\right.$ for 15 minutes) separately from the other medium constituents. Then it is added to the autoclaved brain heart infusion agar with sucrose at $55^{\circ} \mathrm{C}$. CRA plates will be inoculated with test organisms and incubated at $37^{\circ} \mathrm{C}$ for 24 to 48 $\mathrm{h}$ aerobically. Black colonies with a dry crystalline consistency indicate biofilm production.

\section{Biofilm production by tube adherence method}

It is third method done as described by (Christensen et al., 1982) Suspension of tested strains will be incubated in the glass tubes containing Brain Heart Infusion Broth aerobically at the temperature of $35^{\circ} \mathrm{C}$ for a period of two days.

Then the supernatant discarded and the glass tube will be stained by $0.1 \%$.Safranin solution, washed with distilled water three times and dried. A positive result is defined as the presence of a layer of stained material adhered to the inner wall of the tubes. The exclusive observation of a stained ring at the liquid-air interface will be considered negative.

The amount of biofilm formed was scored as 1-weak/none, 2-moderate and 3-high/strong. (Hassan Afreenish et al., 2011).

Fig.1 Proteus species showing non lactose fermenting colonies on MacConkey agar

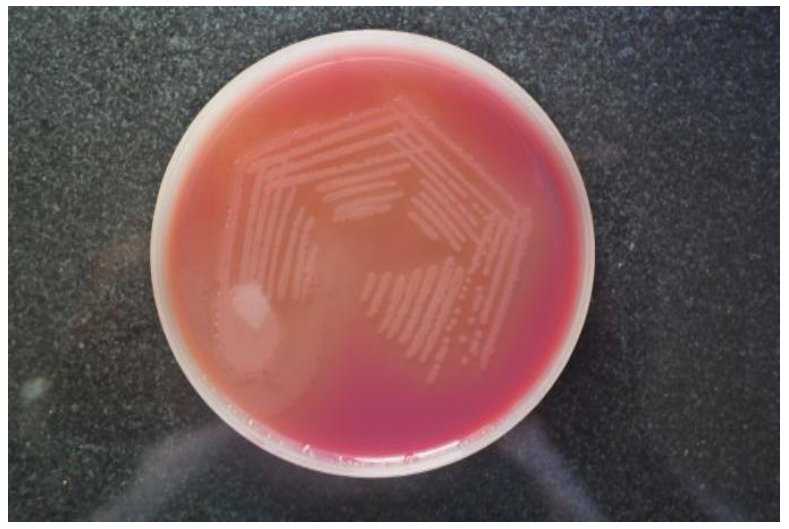


Fig.2 Proteus species negative (left) and positive (right) biofilm producer by Congo red agar method

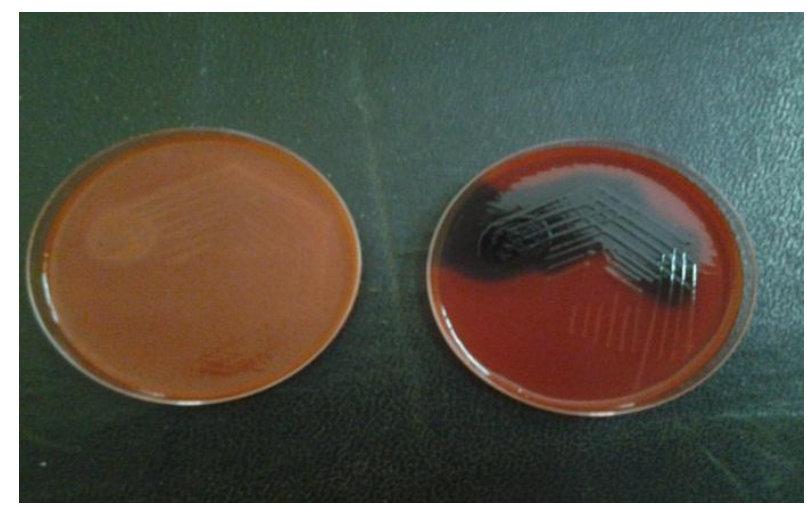

Fig.3 Tube adherence method: left showing 0 (non-biofilm producer), 1 (mild biofilm producer), 2 (moderate biofilm producer), 3(strong biofilm producer) from top to bottom respectively. Right set of tubes are test strains

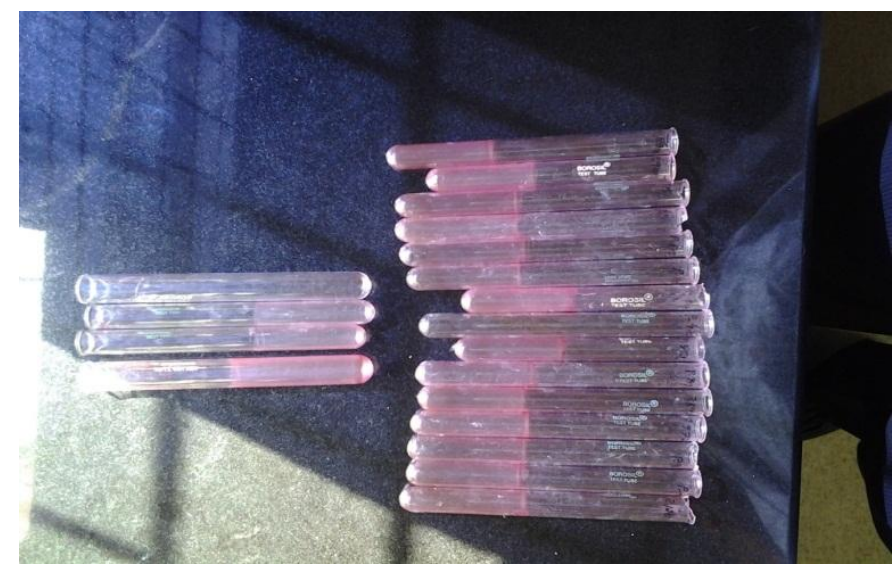

Fig.4 Tissue culture plate method: 1: Strong biofilm producer. 2: Moderate biofilm producer 3: Mild biofilm producer 4: non biofilm producer

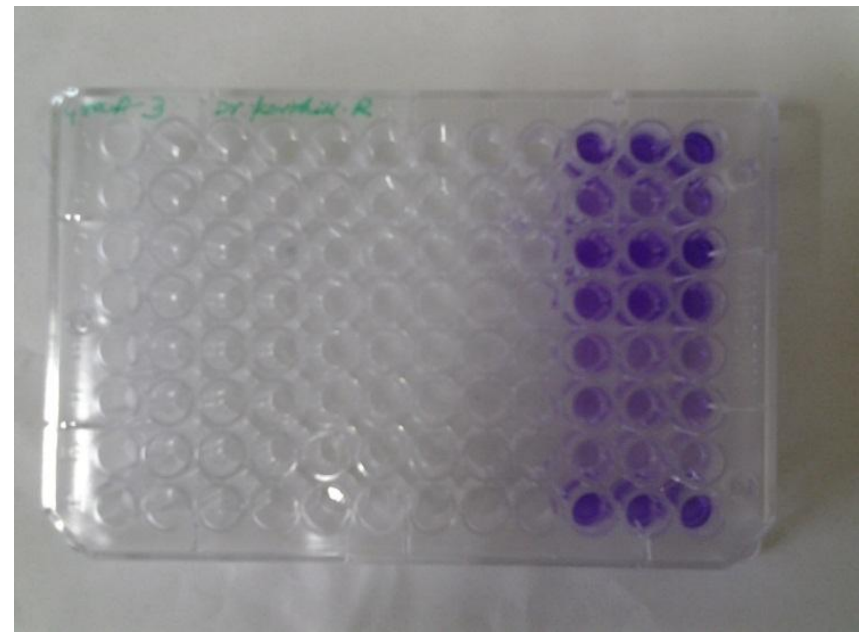


Fig.5 Antibiotic susceptibility pattern of Proteus species isolates

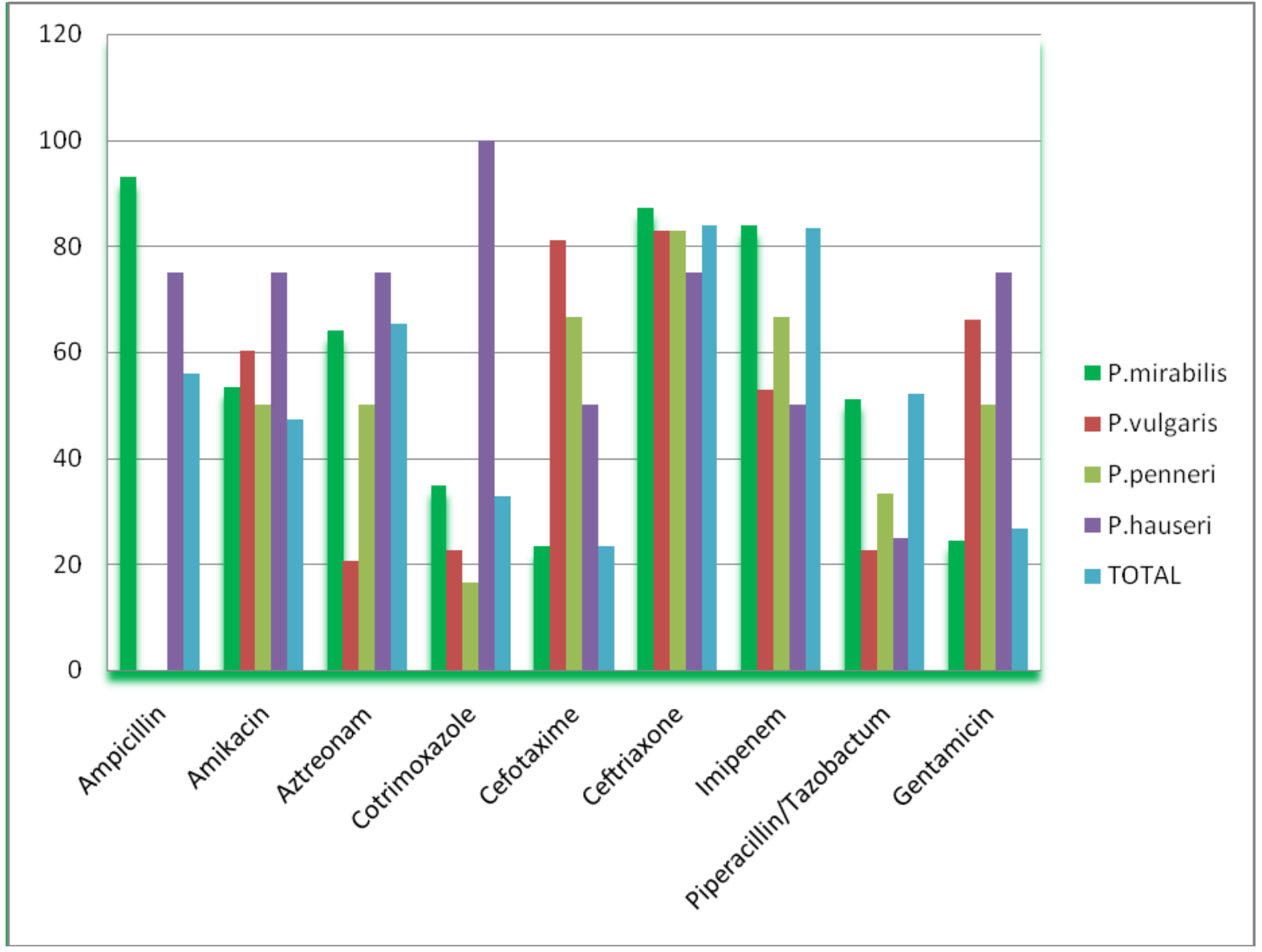

Fig.6 Antimicrobial resistance pattern among biofilm producers and non-producers

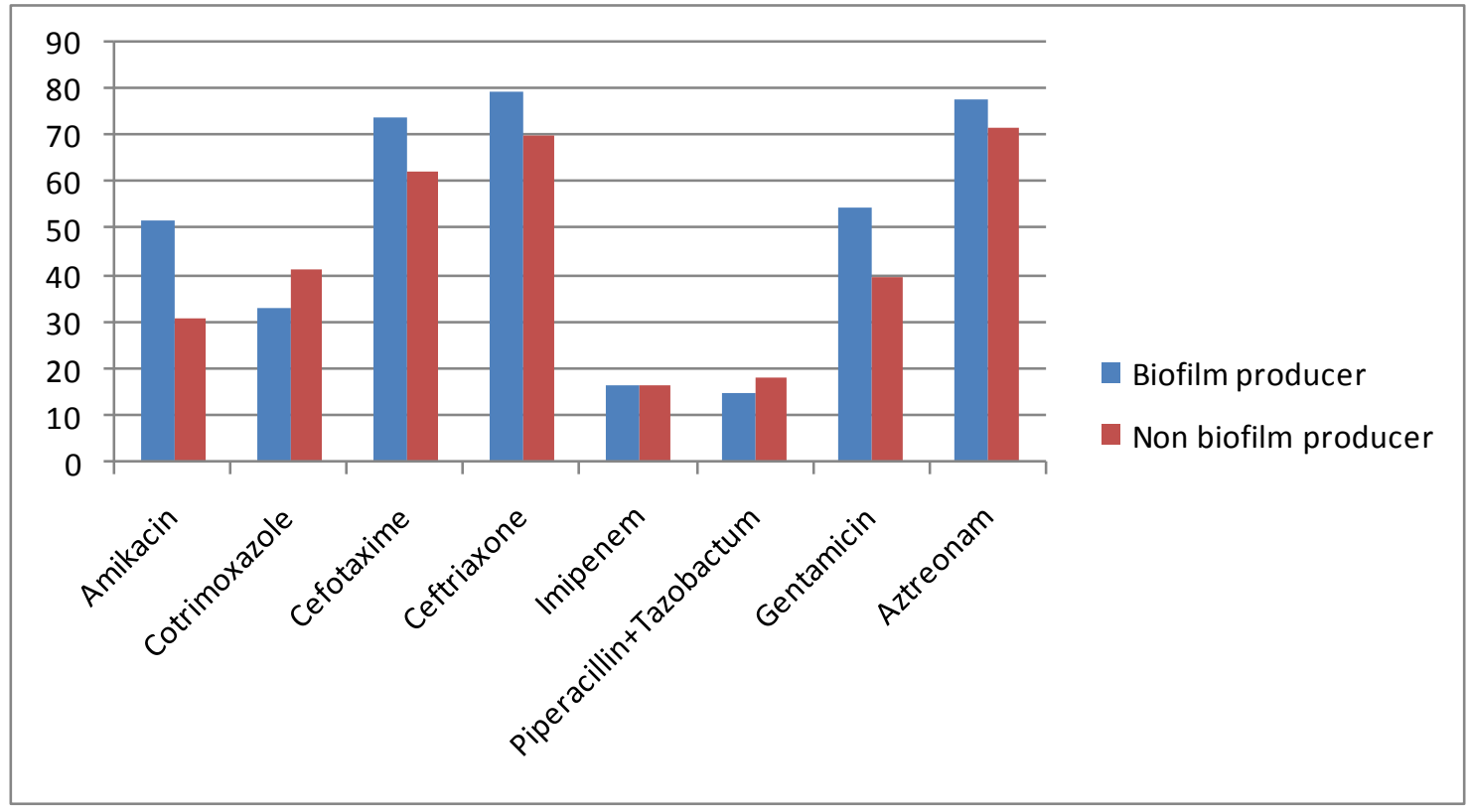


Table.1 Correlation between tube adherence method and tissue culture plate method (Gold standard-Mathur et al., 2006)

\begin{tabular}{|l|l|l|l|l|l|}
\hline Correlating & Positive & Negative & Total & \\
\hline $\begin{array}{l}\text { Not } \\
\text { correlating }\end{array}$ & 16 & 60 & 121 & $\begin{array}{l}\text { Positive predictive value: } \\
81.1 \%\end{array}$ \\
\hline Total & 72 & 18 & 29 & $\begin{array}{l}\text { Negative predictive } \\
\text { value:80.2\% }\end{array}$ & \\
\hline & $\begin{array}{l}\text { True positive rate: } \\
\text { (Sensitivity):77.7\% }\end{array}$ & $\begin{array}{l}\text { False positive } \\
\text { rate: } 23 \%\end{array}$ & $\begin{array}{l}\text { Accuracy: } \\
80.66 \%\end{array}$ & \\
\\
$\begin{array}{lllll}\text { False negative rate: } \\
22.2 \%\end{array}$ & $\begin{array}{l}\text { True negative } \\
\text { rate: } 76.9 \%\end{array}$ & & \\
\hline
\end{tabular}

Table. 2 Correlation between Congo red agar method and tissue culture plate method (Gold standard-Mathur et al., 2006)

\begin{tabular}{|c|c|c|c|c|}
\hline & Positive & Negative & Total & \\
\hline Correlating & 30 & 64 & 94 & $\begin{array}{l}\text { Positive predictive } \\
\text { value: } 68.18 \%\end{array}$ \\
\hline Not correlating & 42 & 14 & 56 & $\begin{array}{l}\text { Negative predictive } \\
\text { value: } 60.3 \%\end{array}$ \\
\hline \multirow[t]{3}{*}{ Total } & 72 & 78 & 150 & \\
\hline & $\begin{array}{l}\text { True positive rate: } \\
\text { (Sensitivity): } 41.6 \%\end{array}$ & $\begin{array}{l}\text { False positive rate: } \\
17.9 \%\end{array}$ & $\begin{array}{l}\text { Accuracy: } \\
64 \%\end{array}$ & \\
\hline & $\begin{array}{l}\text { False negative rate: } \\
58.3 \%\end{array}$ & $\begin{array}{l}\text { True negative rate } \\
\text { (Specificity): } 82.05 \%\end{array}$ & & \\
\hline
\end{tabular}

Table.3 Correlation between Congo red agar method +tube adherence and tissue culture plate method (Gold standard-Mathur et al., 2006)

\begin{tabular}{|l|l|l|l|l|}
\hline Correlating & Positive & Negative & Total & \\
\hline Not correlating & 10 & 36 & 98 & $\begin{array}{l}\text { Positive predictive } \\
\text { value: 59.6\% }\end{array}$ \\
\hline Total & $\begin{array}{l}\text { Negative predictive } \\
\text { value: 78.2\% }\end{array}$ \\
\hline & $\begin{array}{l}\text { True positive rate: } \\
\text { (Sensitivity): } \\
86.11 \%\end{array}$ & $\begin{array}{l}\text { False positive } \\
\text { rate: } 53.8 \%\end{array}$ & $\begin{array}{l}\text { Accuracy: } \\
65.3 \%\end{array}$ & \\
\hline & $\begin{array}{l}\text { False negative rate: } \\
13.8 \%\end{array}$ & $\begin{array}{l}\text { True negative } \\
\text { rate } \\
\text { (Specificity): } \\
46.1 \%\end{array}$ & & \\
\hline & & & & \\
\hline
\end{tabular}




\section{Quality control}

The following international reference strains will be used as controls: Non-biofilm producers Staphylococcus epidermidis ATCC 12228 (negative control). For positive control, Proteus mirabilis strain from study which produced positive result by all three methods was used after cross checking with department of microbiology, ESIC PGIMSR model hospital Rajajinagar, was used.

Considering Tissue culture method as gold standard, (Mathur et al., 2006), tube adherence method showed $80.66 \%$ correlation. Sensitivity was $77.7 \%$ and specificity was $76.9 \%$.

Tube adherence showed good correlation (accuracy) with TCP method (80.66\%) which was better than congo red agar method (64\%) and Congo red and tube adherence method combined.

Sensitivity was highest with Congo red agar method and tube adherence methods combined $(86.11 \%)$.

Tube adherence method showed good sensitivity (77.7\%) when compared with Congo red agar method which showed lowest sensitivity (41.6\%).

Congo red agar method showed better specificity $82.05 \%$ compared to tube adherence method (76.9\%) and CRA and TA combined $(46.1 \%)$.

Tube adherence method had better positive and negative predictive value (81.1 and $80.2 \%$ respectively) compared to other methods (Table 1-3).

Biofilm producer showed more resistance to Amikacin, Cefotaxime, Ceftriaxone, Gentamicin and Aztreonam in comparison to non-biofilm producing isolates. Proteus species are widespread in the environment and are part of the normal flora of the human gastro intestinal tract. Proteus species currently rank third as cause of uncomplicated cystitis, pyelonephritis, and prostatitis particularly when they are hospital acquired.

They have also been implicated in bacteraemia, empyema, osteomyelitis, burn wound infections. Among the various virulence factors of Proteus species, their ability to form biofilm is very important due to the fact that it leads to antimicrobial resistance and treatment failures, chronic infections, embolism and distant site infections. The treatment of Proteus species infections is increasingly becoming difficult because of their ability to form biofilms and multidrug resistance exhibited by these organisms. The results of 150 Proteus species isolates from different clinical cases were studied compared and discussed with the results of other studies.

In the present study majority of the Proteus species isolates were between the age group 20-59 years which correlates with (Feglo et al., 2010) wherein maximum number of Proteus species isolated were between 20-59 years of age. In the present study there is male preponderance. This finding co related with (Saleh et al., 2013). Males were found to be more vulnerable than females in acquiring Proteus species infections. From comparing with other studies it was observed that the present study clearly showed predominance of pus samples from where Proteus species were isolated followed by urine and endotracheal aspirates. (Nita pal et al., 2014) showed similar pattern of isolation of Proteus species in clinical specimens. Proteus seems to be a common cause of wound infections in India and other third world countries in comparison to those from Europe where they cause more urine infections than wound infections. 
Comparison of biofilm production among Proteus species isolates by tissue culture plate method

The therapeutic obstacles during $P$. mirabilis treatment can be connected with its ability to form biofilm. Biofilm is a formation of communicating microorganisms, adhering to certain surfaces and to neighbouring cells, covered with an extracellular matrix. It may consist of one or various species. The ability to form biofilm promotes the development and chronicity of infections. Biofilm can form on non-living as well as living surfaces such as wound surface. Also biofilm organism show increased antimicrobial resistance. Among the Proteus species isolates the present study showed $48 \%$ as biofilm producers and $52 \%$ as biofilm non producers which correlated with other similar study done by Bardsiri Shikh-Bardsiri and Mohammad Reza Shakibaie (2013) who reported $43 \%$ of isolates as non/weak biofilm producers and $57 \%$ as biofilm producers. Among moderate biofilm producers, the present study (29.4\%) correlated with Zubair et al., (2011) (31\%). In the present study tube adherence method showed $77.7 \%$ sensitivity and $76.9 \%$ specificity with accuracy of $80.66 \%$. The sensitivity and accuracy correlated well study by Mathur et al., (2006) which showed $73.6 \%$ sensitivity and $82.7 \%$ accuracy as well as Bose et al., (2009) which showed $76.27 \%$ sensitivity and $81 \%$ accuracy. In the present study, Congo red agar method showed $41.6 \%$ sensitivity and $81.05 \%$ specificity with accuracy of $64 \%$. The present study showed low sensitivity but higher specificity as shown by Mathur et al., (2006) and Bose et al., (2009) in their studies $(6.8 \%$ and $8.25 \%$ sensitivity respectively and $90.2 \%$ and $96.34 \%$ specificity respectively). Accuracy of the present study (64\%) was similar to Bose et al., (2009) (55\%). The findings of the present study indicates high level of multi drug resistant Proteus isolates with Proteus penneri being always multi drug resistant as similar results were found in other studies. The results of Proteus mirabilis (62.7\%) and Proteus vulgaris (67.3\%) correlated with Feglo et al., (2010) 84.6\% and $93.4 \%$ respectively. In the present study, Proteus mirabilis showed more resistance to commonly used antibiotics such as Ceftriaxone, Cefotaxime, Aztreonam. There is also increasing resistance to Gentamicin. High resistance to Cefotaxime $(65.11 \%)$ was seen as reported by others (Donlan and Costerton, 2002). Amikacin resistance was seen in $46.5 \%$ of cases, similar to Jitendra Kumar Pandey et al., (2013) (60\%) and Nita Pal et al., (2014) (65\%). There was high resistance to Aztreonam (75.5\%) which corroborates with earlier observations (Wenner and Rettger, 1919).

In the present study the most sensitive drug is Imipenem with $87.3 \%$. This is correlated with Nita Pal et al., (2014) with $94 \%$ sensitivity. The least sensitive drug is Aztreonam with $24.5 \%$ which corroborated with Jitendra Kumar Pandey et al., (2013). In the present study, Proteus vulgaris showed more resistance to commonly used antibiotics such as Ceftriaxone, Cefotaxime, Aztreonam. There is also increasing resistance to Gentamicin. High resistance to Cefotaxime (79.24\%) was seen which correlated with Nita Pal et al., (2014) Amikacin resistance was seen in $33.9 \%$ of cases, similar to Jitendra Kumar Pandey et al., (2013) (35\%). There was high resistance to Aztreonam (77.3\%) which corroborates with Jitendra Kumar Pandey et al., (2013). In the present study the most sensitive drug is Imipenem with $81.14 \%$.This is correlated with Jitendra Kumar Pandey et al., (2013) with 80\% sensitivity. The least sensitive drug was Cefotaxime $(79.24 \%$ resistance) which correlated with Nita Pal et al., (2014). In the present study, Proteus penneri showed more resistance to commonly used antibiotics such 
as ceftriaxone, Aztreonam. High resistance to ceftriaxone (83.4\%) and Aztreonam (66.67\%) was seen which correlated with Jitendra Kumar Pandey et al., (2013). Amikacin resistance was seen in $50 \%$ of cases, similar to Jitendra Kumar Pandey et al., (2013) (60\%).

Proteus species are important cause of nosocomial infections including uncomplicated cystitis, pyelonephritis, and prostatitis. They have also been implicated in bacteraemia, empyema, osteomyelitis, burn wound infections. They are associated with prolonged hospitalization andthe complications of long-term urinary catheterization. Also their ability to form biofilm is implicated in chronic infections and increased drug resistance. In the present study 150 Proteus species isolates from different clinical conditions were studied for their identification, antibiotic susceptibility pattern and their ability to form biofilm in vitro by three phenotypic methods which included tissue culture plate method, tube adherence method and Congo red agar method.

The majority of Proteus species isolates were between the age group 20-59 yrs. Among the 150 isolates $52.66 \%$ isolates were from male and $47.3 \%$ from females. Among the 150 isolates, $57.3 \%$ were Proteus mirabilis, followed by Proteus vulgaris 36\%, Proteus penneri $4 \%$ and Proteus hauseri $2.7 \%$. Among the total isolates, $48.66 \%$ were biofilm producers and remaining were non biofilm producers. Among the biofilm producers, $38.8 \%$ were strong and $61 \%$ were moderate biofilm producers. Also tube adherence method for biofilm detection showed good correlation with tissue culture plate method and had good sensitivity, specificity and accuracy in comparison to Congo red agar method in biofilm detection. Congo red agar method and tube adherence method in combination showed higher sensitivity in biofilm detection compared to those methods used alone. AST of the isolates showed maximum sensitivity to Imipenem and Piperacillin/tazobactum followed by Cotrimoxazole, Amikacin, Gentamicin, Cefotaxime and least for Ceftriaxone and Aztreonam. Also majority of the isolates were multi drug resistant (MDR) and these MDR isolates showed better biofilm forming capacity than non MDR isolates. Also biofilm producers were more resistant to antimicrobial agents than non-biofilm producers.

The above results clearly points to the medical importance of biofilms. Tube adherence method and Congo red agar methods in combination can be used for screening isolates for biofilm forming ability since tissue culture plate method is more laborious and technically demanding procedure. Also there is an increase in isolation of Proteus species from wounds than from urinary tract infections with significant numbers showing biofilm forming capability and antimicrobial drug resistance indicating need for further studies on Proteus species. Tube adherence method and Congo red agar method in combination proved very effective for screening biofilm forming isolates. Though PCR technique is the most accurate and specific method for detecting the ability to form biofilm, it is not readily available in resource limited settings and the need of the hour is rapid, cheap, cost effective and easily available methods for detecting biofilm formation. However, further studies on better understanding of bacterial biofilm interactions at molecular level for this species are necessary as they are more frequently being implicated in chronic infections and treatment failures. In view of emerging drug resistance and multidrug resistance exhibited by Proteus species, periodic review and formulation of antibiotic policy are needed. Indiscriminate use of antibiotic must be discouraged and 
therapy should be advocated as for as possible after the culture and sensitivity reports are available.

\section{References}

Bose S, Khodke M, Basak S, Mallick Sk. Detection of biofilm producing Staphylococci: Need of the Hour. Journal of clinical and diagnostic research. 2009; 3: 1915-20.

Christensen G.D, Simpson W.A, Bismo A.L, Beachery E.H, Adherence of slimeproducing strains of Staphylococcus epidermidis to smooth surfaces. Infect Immun 1982; 37: 318-326.

Clinical and Laboratory Standard Institute (CLSI). Performance Standards for Antimicrobial Disk Susceptibility Tests: Approved standards- Twelfth edition. CLSI Waine, Pennsylvania. USA. 2012.

Collee JG, Duguid JP, Fraser AG, Marmion BP, Simmons A, Laboratory strategy in the diagnosis of infective syndromes. In: Collee JG, Duguid JP, Fraser AG, Marmion BP, Simmons A editors. Mackie and Mc Cartney Practical Medical Microbiology. $14^{\text {th }}$ edition. Churchill Livingston London. 1996: $53-$ 94.

Donlan RM and Costerton JW 2002. Biofilms: Survival mechanisms of clinically relevant microorganisms. Clinical Microbiology Reviews; 15(2): 167 - 193.

Feglo P.K., Gbedema S.Y., Quay S.N.A., Adu-Sarkodie Y., and C. Opoku-Okrah 2010. Occurrence, species distribution and antibiotic resistance of Proteus isolates: A case study at the Komfo Anokye Teaching Hospital (KATH) in Ghana. International Journal of Pharma Sciences and Research (IJPSR), 1(9): 347-352

Freeman J, Falkiner FR, Keane CT. New method for detecting slime production by coagulase negative Staphylococci. J Clin Pathol 1989; 42:872-4.

Hassan Afreenish, Usman Javaid, Kaleem Fatima, Omair Maria, Khalid Ali, Iqbal Muhammad. Evaluation of different detection methods of biofilm formation in the clinical isolates. Braz J Infect Dis [serial on the Internet]. 2011 Aug [cited 2014 Nov 06] ; 15(4): 305-311

Houshang Shikh-Bardsiri and Mohammad Reza Shakibaie, 2013. Antibiotic Resistance Pattern among Biofilm Producing and Non-Producing Proteus Strains Isolated from Hospitalized Patients; Matter of Hospital Hygiene and Antimicrobial Stewardship. Pakistan Journal of Biological Sciences, 16: 1496-1502.

Jitendra Kumar Pandey, Akanksha Narayan and Shikhar Tyagi Prevalence of Proteus species in clinical samples, antibiotic sensitivity pattern and ESBL production

Int.J.Curr.Microbiol.App.Sci.

2013.2(10): 253-261

Joanna Kwiecińska-Piróg, Krzysztof Skowron, Katarzyna Zniszczol, and Eugenia Gospodarek, "The Assessment of Proteus mirabilis Susceptibility to Ceftazidime and Ciprofloxacin and the Impact of These Antibiotics at Subinhibitory Concentrations on Proteus mirabilis Biofilms," BioMed Research International, vol. 2013, Article ID 930876, 8 pages, 2013. doi:10.1155/2013/930876

Longo Dan. L, Kasper Dennis.L, Fauci Anthony. S, Hauser Stephen.L, Loscalzo Joseph. L. Harrison's principles of internal medicine $18^{\text {th }}$ ed 2011, part 8, section 6 chapter 149.

Mandell Gerald L, Bennett John E, Rapahel Dolin. Section A, Chapter 2. Microbial adherence, Microbial biofilms. Mandell, Douglas and Bennett's Principles and Practice of Infectious diseases. $7^{\text {th }}$ ed. $\mathrm{P}$ 
22-23.

Mathur T, Singhal S, Khan S, Upadhyay D J, Fatma T, Rattan A. Detection of biofilm formation among the clinical isolates of Staphylococci: An evaluation of three different screening methods. Indian $\mathrm{J}$ Med Microbiol 2006; 24:25-9

Nita Pal, Nikita Sharma, Rajni Sharma, Saroj Hooja, and Rakesh K Maheshwari 2014. Prevalence of Multidrug (MDR) and Extensively Drug Resistant (XDR) Proteus species in a tertiary care hospital, India Int.J.Curr.Microbiol. App.Sci. 2014.3(10): 243-252

Saleh A. Bahashwan, Hatem M. El Shafey, European Scientific Journal September 2013 edition vol.9, Antimicrobial resistance patterns of Proteus isolates from clinical specimens. European Scientific Journal September 2013 edition vol.9, No.27 ISSN: $1857-7881$ (Print) e -ISSN 1857-7431

Stepanović S, Vuković D, Hola V, Di Bonaventura G, Djukić S, Cirković I, Ruzicka F. Quantification of biofilm in microtiter plates: overview of testing conditions and practical recommendations for assessment of biofilm production by Staphylococci. APMIS 2007; 115: 891-9.

Stoodley L, Costerton JW, Stoodley P, 2004. Bacterial biofilms: from the natural environment to infectious diseases. Nature Reviews Microbiology, 2 (2): 95-108.

Wenner, J.J., and L.F. Rettger. 1919. A systematic study of the Proteus group of bacteria. Journal of Bacteriology 4(4) (Jul):331-53.

Zubair M, Malik A, Ahmad J, Rizvi M, Farooqui KJ, Rizvi MW. A Study of biofilm production by gram negative organisms isolated from diabetic foot ulcer patients. Biology and Medicine, 3(2) Special issue: 147-157, 2011.

Zubair M, Malik A, Ahmad J, Rizvi M, Farooqui KJ, Rizvi MW. A Study of biofilm production by gram negative organisms isolated from diabetic foot ulcer patients. Biology and Medicine, 3(2) Special issue: 147-157, 2011.

\section{How to cite this article:}

Karthik, R., R. Ambica and Nagarathnamma, T. 2018. Study of Biofilm Production and Antimicrobial Susceptibility Pattern in Clinical Isolates of Proteus Species at a Tertiary Care Hospital. Int.J.Curr.Microbiol.App.Sci. 7(01): 574-586.

doi: https://doi.org/10.20546/ijcmas.2018.701.070 